\title{
Riscos ocupacionais em profissionais de enfermagem de uma Unidade de Terapia Intensiva adulta, localizada em um município de Pernambuco
}

\author{
Occupational risks in nursing professionals in an adult Intensive Care Unit, located in a \\ municipality of Pernambuco
}

Riesgos laborales en profesionales de enfermería en una Unidad de Cuidados Intensivos para adultos, ubicada en un municipio de Pernambuco

Suellen Alyne Alves dos Santos ${ }^{1 *}$, Sheila Juliana Leite Lima1, Daniela de Aquino Freire ${ }^{2}$, Andreza Cavalcanti Vasconcelos ${ }^{1}$, Sâmara Aline Brito Brainer ${ }^{1}$, Clícia Meyriele de Oliveira Bezerra ${ }^{1}$, Ana Paula dos Santos Albuquerque ${ }^{2}$, José Osmar da Silva ${ }^{1}$, Hanna Carolina Padilha de Siqueira ${ }^{3}$, Roberto dos Santos Siqueira ${ }^{4}$.

\section{RESUMO}

Objetivo: Identificar os riscos ocupacionais que profissionais de enfermagem estão expostos atuando em uma UTI adulto de um hospital regional em um município de Pernambuco. Métodos: Trata-se de um estudo descritivo com abordagem quantitativa. A amostra foi composta por 39 profissionais de enfermagem. Utilizouse de um questionário semiestruturado e adaptado no período de fevereiro a março de 2018. Resultados: A idade média da população foi de $36,82 \pm 8,36$ anos, $76,92 \%$ eram do sexo feminino, $100 \%$ dos profissionais acreditavam que o ambiente de trabalho trazia risco para sua saúde, demonstrando que entre as categorias de saúde, a enfermagem é a mais exposta aos riscos ocupacionais. Entre os entrevistados, 35,90\% tiveram acidentes no seu local de trabalho. Conclusão: $O$ ambiente laboral pode levar os profissionais à exposição de diversos riscos, necessitando de adoção de medidas de biossegurança e precaução padrão, de modo a minimizar os riscos de acidentes ou doenças relacionadas ao trabalho.

Palavras-chave: Riscos ocupacionais, Unidade de Terapia Intensiva, Acidentes de trabalho.

\section{ABSTRACT}

Objective: To identify the occupational risks that nursing professionals are exposed working in an adult ICU of a regional hospital in a municipality in Pernambuco. Methods: This is a descriptive study with a quantitative approach. The sample consisted of 39 nursing professionals. A semi-structured questionnaire was used and adapted from February to March 2018. Results: The average age of the population was $36.82 \pm 8.36$ years, $76.92 \%$ were female, $100 \%$ of professionals believed that the work environment brought risk to their health, demonstrating that among health categories, nursing is the most exposed to occupational risks. Among the interviewees, $35.90 \%$ had accidents at their workplace. Conclusion: The work environment can lead professionals to exposure to various risks, requiring the adoption of biosafety measures and standard precautions, in order to minimize the risks of accidents or illnesses related to work.

Keywords: Occupational risks, Intensive Care Unit, Accidents at work.

${ }^{1}$ Centro Universitário Vale do Ipojuca (UNIFAVIP), Caruaru - PE. *E-mail: suellenalynee@gmail.com

2 Universidade Estadual de Pernambuco (UPE), Caruaru - PE.

3 Universidade Estadual de Pernambuco (UPE), Garanhuns - PE.

4 Universidade Federal de Pernambuco (UFPE), Recife - PE. 


\section{RESUMEN}

Objetivo: Identificar riesgos laborales en profesionales de enfermería que laboran en una UCI de adultos de un hospital regional de un municipio de Pernambuco. Métodos: Se trata de un estudio descriptivo con enfoque cuantitativo. La muestra estuvo formada por 39 profesionales de enfermería. Se utilizó un cuestionario semiestructurado adaptado de febrero a marzo de 2018. Resultados: La edad promedio de la población fue de 36,82 $\pm 8,36$ años, el 76,92\% eran mujeres, el 100\% de los profesionales consideró que el entorno de Este trabajo trajo riesgos para su salud, mostrando que entre las categorías de salud, la enfermería es la más expuesta a riesgos laborales. Entre los entrevistados, el 35,90\% sufrió accidentes laborales. Conclusión: El ambiente de trabajo puede llevar a los profesionales a la exposición a diversos riesgos, requiriendo la adopción de medidas de bioseguridad y precauciones estándar, a fin de minimizar los riesgos de accidentes o enfermedades relacionadas con el trabajo.

Palabras clave: Riesgos laborales, Unidad de Cuidados Intensivos, Accidentes de trabajo.

\section{INTRODUÇÃO}

A Unidade de Terapia Intensiva (UTI) é um local destinado às pessoas que requerem cuidados que difere das demais unidades de internação, devido à necessidade de cuidado de moderada a alta complexidade, com tratamento considerado mais intensivo e invasivo (AIRES RKD, et al., 2020). Por conseguinte, as exigências impostas nessas unidades podem superar o limite do profissional, induzindo-o ao estresse prolongado que podem emergir sofrimentos relacionados à sua história individual. Os desgastes neste ambiente podem implicar no surgimento de diversas doenças (SANTOS SS, et al., 2018).

O enfermeiro intensivista demanda conhecimentos específicos à utilização de materiais de insumos para procedimentos, que aumentam o risco de exposição em um ritmo de atividade intensa, tanto que os riscos ocupacionais nos trabalhadores de enfermagem estariam relacionados com o cuidado e às próprias características dos pacientes críticos, como a presença de sangue, procedimentos de sondagem, cateterismo e intervenções com materiais perfurocortantes. Devido aos riscos de exposição, durante a assistência direta, foi aplicado ao meio laboral a Norma Regulamentadora 32 (NR-32) com a finalidade de estabelecer diretrizes básicas à implementação de medidas de proteção à segurança dos trabalhadores nos serviços de saúde, bem como os que exercem atividades de promoção e assistência em geral nas instituições de saúde (MORAES KKO, et al., 2016)

Nesse sentido, o ambiente laboral é o local em que o trabalhador passa grande parte do seu dia, exercendo suas atividades em contato com diversos pacientes, com inúmeras enfermidades, gerando grande influência sobre sua saúde física e mental. A saúde ocupacional, ou saúde do trabalhador, está associada diretamente à estabilização da integridade física e mental do trabalhador, bem como à promoção da saúde durante o exercício do trabalho. O enfermeiro que atua no ambiente hospitalar está exposto ao desgaste mental, físico e emocional, a sobrecarga de trabalho, a quantidade insuficiente de profissionais e instalações físicas, por vezes, inadequadas. Levando assim a exposição a diversos riscos laborais (OLIVEIRA MM, et al., 2017).

Percebe-se uma carência no papel exercido pelos trabalhadores de enfermagem, pois esses estão trabalhando em inúmeras situações de risco, em diversos ambientes hospitalares e em setores críticos como é o caso da Unidade de Terapia Intensiva, entre outros, levando-os, muitas vezes, ao processo de adoecimento. Diante disso, esses profissionais estão vulneráveis a praticarem atos de negligência, imperícia e imprudência, que estão associados às práticas instáveis no exercício do seu ofício. Nesse contexto, por vezes, esses profissionais também lidam com a ausência de suporte por parte dos gestores, podendo afetar diretamente a saúde do trabalhador, devido a falta de condições de prestar uma assistência com qualidade e segurança para esses profissionais. Um fator de grande relevância é a falta de equipamentos de proteção individual, que, muitas vezes, a gestão não fornece materiais suficientes para a demanda apresentada, porém é sabido que é um direito de cada profissional estar devidamente provido de tais materiais (FONTANA e LAUTERT, 2013). 
Diante desse contexto, os profissionais de enfermagem mantêm uma maior aproximação com pacientes e familiares, estando envolvidos com os aspectos emocionais, estresses e outros sentimentos apresentados pelos familiares dos pacientes. Além disso, a demanda advinda do exercício profissional, como necessidade de atualizações, capacitações, diferentes condições de trabalho, novas configurações organizacionais, podem provocar, consequentemente, o desgaste físico, psíquico e emocional (AIRES RKD, et al., 2020).

A maior incidência de acidentes ocorridos com a equipe de enfermagem está relacionada à perfuração com materiais cortantes $(60,7 \%)$, devido, principalmente, ao manuseio de agulhas e à utilização de bisturis. Além disso, descarte de perfurocortantes em locais inadequados ou em recipientes que já excederam seu limite, a manipulação de agulhas desprotegidas, a desconexão da agulha da seringa e o reencape de agulhas são consideradas causas para esse tipo de acidente. Os profissionais de saúde, por vezes, preocupam-se mais com a assistência oferecida aos usuários do que com os riscos inerentes à execução de suas atividades, podendo-se perceber ainda o excesso de autoconfiança adquirida com os anos de experiência profissional. No entanto, os acidentes de trabalho são, algumas vezes, ignorados e não notificados devido ao desconhecimento da obrigatoriedade do procedimento, a não caracterização do episódio como acidente e ao medo do trabalhador acidentado de realizar a notificação e, consequentemente, ser punido (MELO FMS, et al., 2017).

Dessa forma, o objetivo do estudo foi identificar os riscos ocupacionais em profissionais de enfermagem que atuam em uma UTI adulto de um hospital regional em um município de Pernambuco.

\section{MÉTODOS}

Trata-se de um estudo descritivo com abordagem quantitativa realizado em uma UTI adulto de um Hospital Regional localizado no interior de Pernambuco, com 39 profissionais de enfermagem, sendo 09 enfermeiros e 30 técnicos de enfermagem.

Para escolha da amostra foram considerados os seguintes critérios de inclusão: ser enfermeiro ou técnico de enfermagem; atuar na UTI e concordar em assinar o Termo de Consentimento Livre e Esclarecido (TCLE). Foram excluídos os profissionais de enfermagem que estavam em período de férias ou de licença médica durante a realização da pesquisa.

Para coleta de dados foi utilizado um questionário semiestruturado e adaptado do estudo (CANALLI RTC, 2012) contendo 20 perguntas referentes ao perfil sociodemográfico dos profissionais, como idade, sexo, categoria profissional, tempo de atuação na UTI, turno e especialização, além de variáveis envolvendo os riscos ocupacionais como ruídos, exposição ao uso contínuo de antibióticos, contato com sangue e microorganismos, esforço físico, risco de acidentes, sobrecarga de trabalho física excessiva, ambiente estressante, acidentes no local de trabalho, utilização dos equipamentos de proteção individual (EPIs) e atendimento pósexposição, que interferiam na saúde do profissional.

A pesquisa foi iniciada após a emissão da carta de anuência fornecida pelo hospital do estudo e após aprovação do projeto de pesquisa pelo Comitê de Ética em Pesquisa em Seres Humanos do Centro Universitário do Vale do Ipojuca - UNIFAVIP, no que concerne aos aspectos éticos legais de acordo com a Resolução № 466/2012 e 510/2016 do Conselho Nacional de Saúde (CNS), sob o parecer $n^{\circ} 2.476 .318$ (BRASIL, 2012 e BRASIL, 2016).

A coleta de dados foi realizada nos meses de fevereiro e março de 2018. Foi agendada visita prévia à coordenação da UTI, para apresentação da relevância da pesquisa e entrega do projeto. Nesse momento, também foi solicitado informações sobre o melhor horário de acesso à equipe de enfermagem, para que a coleta de dados fosse programada. O profissional que participou do estudo foi direcionado para uma sala previamente reservada no próprio hospital, respeitando a sua privacidade.

Todos os participantes foram claramente informados sobre os objetivos do estudo e da natureza confidencial das informações coletadas. Foi enfatizado seu direito de envolvimento voluntário na pesquisa e que eles podiam recusar a continuidade da entrevista a qualquer momento. Foram garantidos a privacidade, 
confidencialidade, anonimato e o respeito aos valores socioculturais de cada um. Salienta-se que o questionário somente foi aplicado após assinatura do TCLE pelos participantes, em duas vias, ficando uma das vias com o pesquisador e a outra com o funcionário, na qual constou a assinatura do pesquisador.

Os questionários devidamente preenchidos foram submetidos à revisão manual para avaliar à qualidade das informações. Para análise dos dados foi construído um banco no software Excel da Microsoft $2010^{\circledR}$, onde foi realizada a validação (a técnica de dupla digitação foi empregada com a finalidade de detectar possíveis erros lógicos e inconsistências nos registros). Após a validação, o banco de dados foi exportado para 0 software SPSS, versão 21 , no qual foi realizada a análise do perfil sociodemográfico dos funcionários selecionados. Os dados categóricos foram apresentados em frequências absolutas e relativas e sendo expostos através de tabelas e gráficos.

\section{RESULTADOS}

A amostra do estudo foi constituída por 39 profissionais de enfermagem de ambos os sexos. A idade média dos participantes foi de $36,82 \pm 8,36$. A Tabela 1 ilustra o perfil dos profissionais de enfermagem quanto ao sexo, categoria profissional, tempo de atuação na UTI, turno e especialização profissional.

Tabela 1 - Perfil dos profissionais de enfermagem segundo sexo, categoria profissional, tempo de atuação na UTI, turno e especialização profissional.

\begin{tabular}{lcc}
\hline Sexo & $(\mathbf{N})$ & $\%$ \\
\hline Feminino & 30 & 76,92 \\
Masculino & 09 & 23,08 \\
Total & 39 & 100,00 \\
\hline Categoria profissional & $\mathbf{( N )}$ & $\%$ \\
\hline Técnico de Enfermagem & 30 & 76,92 \\
Enfermeiro & 09 & 23,08 \\
Total & 39 & 100,00 \\
\hline Tempo de atuação na UTI & $\mathbf{( N )}$ & $\%$ \\
\hline $1-5$ anos & 29 & 74,35 \\
$6-10$ anos & 06 & 15,38 \\
$11-16$ anos & 04 & 10,24 \\
Total & 39 & 100,00 \\
\hline Turno & $\mathbf{( N )}$ & $\%$ \\
\hline Diurno e noturno & 39 & 100,00 \\
\hline Especialização profissional & $\mathbf{( N )}$ & $\%$ \\
\hline UTI & 18 & 46,15 \\
Outras & 15 & 38,46 \\
Nenhuma & 06 & 15,38 \\
Total & 39 & 100,00 \\
\hline
\end{tabular}

Fonte: Santos SAA, et al., 2020.

Os resultados apresentaram que $100 \%(n=39)$ dos profissionais de enfermagem consideram o seu ambiente de trabalho um fator de risco para sua saúde. Com relação aos riscos ocupacionais, o ruído que está relacionado ao risco físico, obteve $40,74 \%$ (n=33) em relação aos demais. Os químicos representaram $67,27 \%(n=37)$ sendo associados aos antibióticos de forma rotineira. No que se referem aos riscos biológicos, os micro-organismos representaram $28,24 \%(n=37)$ e contato com sangue $28,24 \%(n=37)$ foram os mais relatados pelos profissionais. O esforço físico $31,03 \%(n=36)$ foi o que mais prevaleceu entre o risco ergonômico e o risco de acidentes, 33,33\% $(n=36)$ citaram o material perfurocortante como o mais agravante.

Quanto aos fatores psicossociais 8,37\% $(n=18)$ mencionaram a sobrecarga de trabalho físico excessiva e $14,88 \%(n=32)$ consideram o ambiente estressante. Entre os trabalhadores entrevistados $35,90 \%(n=14)$ sofreram acidentes no seu local de trabalho, enquanto $64,10 \%(n=25)$ nunca tiveram. 
Em relação aos equipamentos de proteção individual (EPIs), 30,77\% ( $n=12)$, estavam utilizando luvas de procedimento no momento do acidente, $30,77 \%(n=12)$ faziam uso de jaleco e 2,56\% $(n=1)$ óculos de proteção. Sobre a notificação após o acidente $10,26 \%(n=4)$ realizaram o preenchimento da Comunicação de Acidentes de Trabalho - CAT. Relacionado ao atendimento pós-exposição 15,38\% $(n=6)$ realizaram exames laboratoriais no paciente-fonte.

O Gráfico 1 abaixo ilustra a distribuição dos profissionais de enfermagem quanto à presença dos riscos ocupacionais na UTI do estudo.

Gráfico 1 - Distribuição dos profissionais de enfermagem quanto à presença dos riscos ocupacionais na

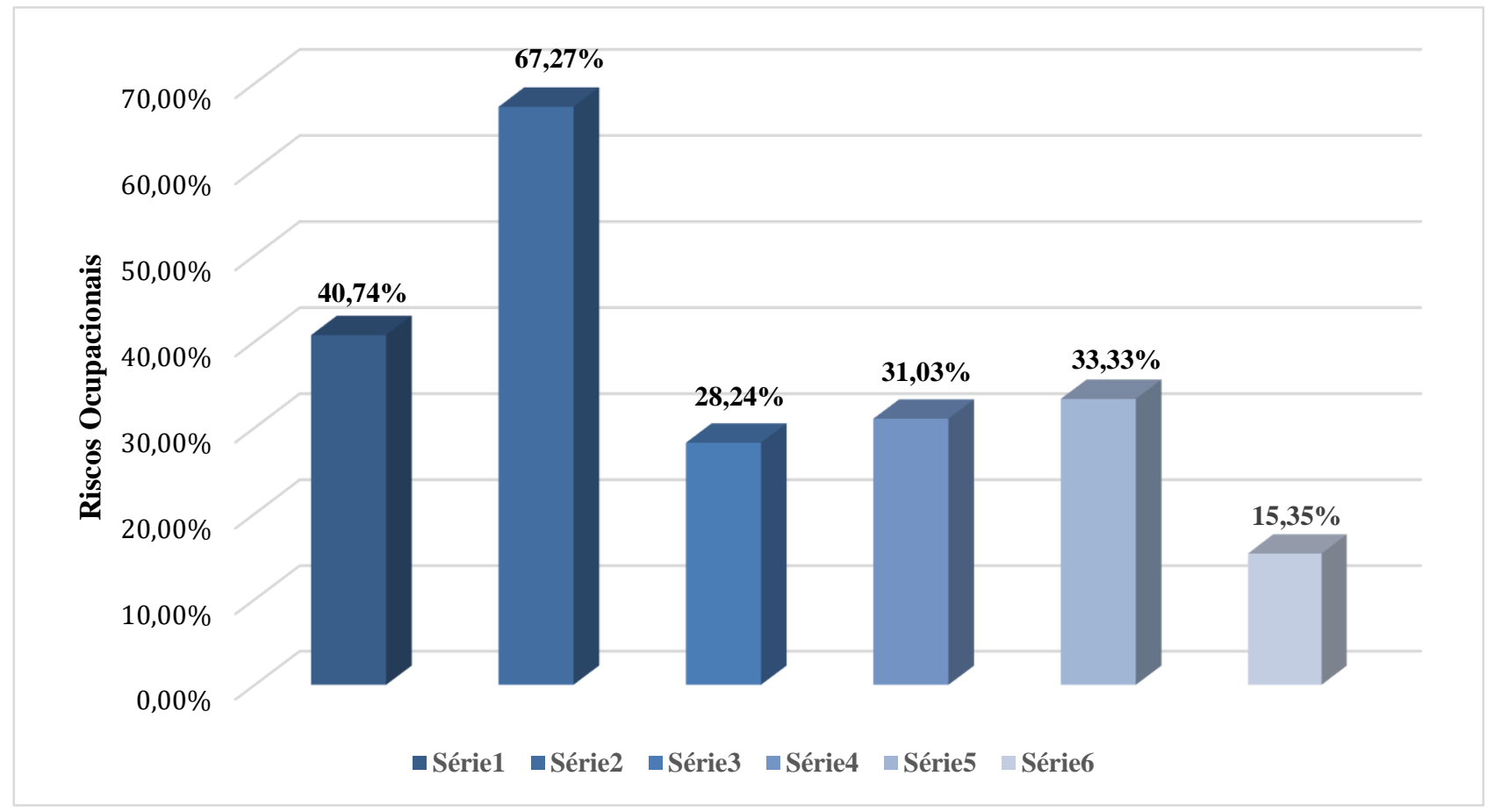

UTI.

Fonte: Santos SAA, et al., 2020.

\section{DISCUSSÃO}

Os resultados relacionados ao perfil sociodemográfico dos profissionais de enfermagem a respeito da idade foi ao encontro de uma pesquisa de Balsanelli AP e Cunha ICKO (2015), na qual mostrou a idade média desses trabalhadores, sendo os técnicos de enfermagem, que atuam em UTI, de 34,7 anos e os enfermeiros apresentou uma média de 32,9, demonstrando que os profissionais de enfermagem são indivíduos adultos jovens.

Em se tratando de informações presentes na tabela, quanto a variável sexo, os dados entram em convergência com um estudo realizado por Pimenta AM e Assunção AA (2016), no qual foi visto que a maioria $90,5 \%$ dos participantes era do sexo feminino, evidenciando uma primazia em relação ao sexo masculino. Isso pode ser explicado devido à profissão de enfermagem ainda ser constituída, na sua integralidade, por mulheres, no qual o ato de cuidar pode estar associado ao extinto da maternidade quando comparado ao sexo masculino.

No quesito dimensionamento, verifica-se que, quando esta etapa não é respeitada, ela favorece a presença de alguns riscos ocupacionais, riscos que são vistos como um ponto negativo. O dimensionamento deve seguir o que a RDC 26/2012, junto à resolução do Conselho Federal de Enfermagem COFEN 527/2016 preconiza sobre o dimensionamento do pessoal da enfermagem, sendo 01 enfermeiro plantonista para cada 10 leitos e mais um enfermeiro diarista e um técnico de enfermagem para cada 02 leitos de UTI mais 01 
técnico diarista (BRASIL, 2016b). Esse dimensionamento está relacionado ao serviço de saúde, aos serviços de enfermagem e a segurança do paciente, bem como ao quantitativo mínimo exigido de profissionais nas 24 horas de plantão pela dependência de cuidados intensivos, intermediários, cuidados de alta dependência e cuidados mínimos (BRASIL, 2016a).

Sobre as categorias de enfermagem, em um estudo realizado verificou que o dimensionamento dos trabalhadores de enfermagem que atuavam em uma UTI de uma instituição de saúde era composta por uma de enfermeiros e técnicos de enfermagem, corroborando com a atual pesquisa (RODRIGUES MA, et al., 2017). Nesse contexto, é de fundamental importância pontuar que o enfermeiro, como responsável por sua equipe, deve ficar atento quanto à estimação e bem estar de todos os profissionais, tendo em vista proteger o trabalhador de riscos ocupacionais e evitando o absenteísmo, que pode gerar uma sobrecarga e prováveis adoecimentos dos trabalhadores.

Em relação ao turno de trabalho, um estudo verificou que $50 \%$ dos profissionais que operavam na UTI trabalhavam em horário diurno e também em horário noturno o que contradiz com esta pesquisa, no qual foi observado que todos os participantes trabalhavam em regime de plantões de 24 horas. Esse dado é bem relevante ao mostrar que o profissional de enfermagem pode encontrar-se exposto a uma maior sobrecarga de trabalho, o que pode influenciar de modo direto na qualidade da assistência prestada a clientela (PADILHA KG, et al., 2017).

No que compete ao tempo de laboração na UTI, foi verificado em um estudo que parte dos profissionais tinha pouco tempo de atuação na área. Esse dado vai ao encontro com o presente estudo, o qual evidencia o quanto o tempo de atuação pode influenciar na maneira que os trabalhadores fornecem a assistência, visto que, o profissional como principiante, pode não possuir um domínio completo do que deve ser cumprido na rotina do setor para efetuar de forma segura (PASCOAL KPMF, et al., 2019).

Sobre a especialização profissional, verificou-se em um estudo que $70 \%$ dos enfermeiros possuíam pósgraduação em UTI, corroborando com os achados da pesquisa, no qual pode ser explicado pelo fato da UTI exigir profissionais mais qualificados, uma vez que é um setor crítico, de maior complexidade e que requer uma maior habilidade e aptidão por parte destes profissionais que exercem nessa área. Nesse sentido, observou-se a necessidade de capacitações, treinamentos sobre os riscos ocupacionais mensalmente para toda a equipe de enfermagem a fim de mantê-los atualizados, constantemente, afim de minimizar riscos ocupacionais e prejuízos relacionados à assistência à saúde (SILVA C, BATISTA EC, 2017).

Com relação ao ambiente de trabalho, um estudo verificou que a enfermagem é a profissão mais exposta aos ricos ocupacionais, pois prestam assistência de forma contínua ao paciente e estão mais susceptíveis a adquirir diversos tipos de enfermidades (RODRIGUES MA, et al., 2017).

Quanto aos riscos físicos, estudo de Christofel HK, et al. (2016) verificou que o ruído é considerado um meio que afeta a saúde de todos corroborando com o presente estudo. Isso pode estar associado ao número de aparelhos e sons transmitidos de forma ininterrupta que podem acarretar modificações na comunicação, perturbações, estresse ou possíveis adoecimentos no trabalho.

No que refere ao risco químico, uma pesquisa constatou que o manejo com substâncias químicas, como antibióticos, é existente na prática dos profissionais de enfermagem, assim como foi analisado no presente estudo. Este risco é visto como prejudicial para a saúde dos trabalhadores, o qual pode gerar alguma doença alérgica e até o desenvolvimento de doença mais grave, como o câncer (PORTELA NLC, et al., 2015).

Dentre os riscos biológicos, foi constatado que o contato com micro-organismos ,tal como bactérias e vírus, é rotineiro entre os profissionais de enfermagem, corroborando com o estudo realizado por Ribeiro IAP, et al. (2017), o qual traz que devido a UTI ser vista como um espaço insalubre, onde os mesmos estão expostos a obter inúmeras doenças, sobretudo, as infectocontagiosas. Ainda sobre os riscos biológicos, um estudo realizado por Rodrigues MA, et al. (2017) atestou -se que o manuseamento com sangue $84,2 \%$ ocorre, em grande parte, nos procedimentos feitos com o paciente, devido a materiais infectados ou uso inadequado dos mesmos, dado este, indo ao encontro com a pesquisa. 
Em estudo realizado por Pasa TS, et al. (2015), observou-se que o esforço físico 56,9\% estava presente na rotina da equipe de enfermagem na unidade de terapia intensiva (UTI), corroborando com o estudo. Dentre as diversas explicações, o esforço físico pode estar relacionado ao fato da equipe lidar rotineiramente com pacientes acamados e que necessitam de movimentação constante e/ou remoção dos mesmos, o que pode favorecer o aumento na probabilidade de doenças osteomusculares.

Em se tratando de risco de acidentes, pôde-se verificar em estudo realizado que cerca de $53,9 \%$ dos trabalhadores relataram o uso inadequado de materiais perfurocortantes. Esse achado vai de encontro com a pesquisa, pois mostra que a exposição do profissional pode levar a ocorrência de acidentes e doenças infecciosas como Hepatite B e HIV, devido o contato com agulhas e seringas contaminadas em procedimentos invasivos (ARAGÃO JÁ, et al., 2019).

Quanto aos riscos psicossociais, estudo demonstrou que a maioria dos participantes relataram que a sobrecarga de trabalho física é devido ao ritmo excessivo de trabalho, levando a um desgaste e desmotivação profissional (VASCONCELOS EM, MARTINO MMF, 2017). Já, com relação ao estresse profissional, estudo constatou que $77 \%$ dos profissionais consideraram o trabalho na UTI um fator estressante, pois cuidam diretamente de pacientes graves, que necessitam de atenção contínua e que estão em situações de sofrimento, afetando sua saúde psicológica e mental, corroborando com os achados da presente pesquisa (CARRACA GLR, et al., 2015).

Em relação ao uso correto dos EPIs na rotina dos profissionais da UTI, esses são medidas de prevenção e proteção ao trabalhador. Identificou-se em um estudo que no momento do acidente os equipamentos mais utilizados são as luvas de procedimentos $92,4 \%$, enquanto óculos de proteção $38,6 \%$ é menos utilizado, resultado que vai de encontro com os dados do estudo. É importante ressaltar a importância do uso dos EPIs de forma correta para evitar acidentes de trabalho (ILAPA-RODRIGUEZ EO, et al., 2018).

Sobre os funcionários que sofreram acidentes no local de trabalho, o estudo corroborou com pesquisa a qual afirmou que os profissionais que trabalham na UTI são mais vulneráveis aos riscos ocupacionais por se tratar de um setor estressante e conflitante, também por contato com diversas doenças e procedimentos que são realizados com pacientes em estado grave (MORAES KKO, et al., 2016).

Ainda se tratando de acidente, uma pesquisa mostrou que menos da metade daqueles que sofreram esse dano fizeram a notificação e preenchimento da CAT, o que foi ao encontro com os achados da presente pesquisa. Tendo em vista que a notificação é desconhecida pela maioria dos trabalhadores, só que é uma atividade obrigatória que vem sendo negligenciada por parte dos profissionais da área da saúde. É preciso conscientizar os trabalhadores quanto à necessidade e importância da sua notificação (LUIZE PB, et al., 2015).

Ademais, sobre o atendimento pós-exposição, estudos mostraram que $86,8 \%$ dos acidentados efetuaram exames laboratoriais no paciente fonte, pós exposição. Esse achado corrobora com o estudo, no qual somente alguns dos participantes realizaram o teste no paciente, após o acidente. É importante priorizar a realização do teste, pois é possível identificar se foi reagente e a possibilidade de iniciar o tratamento para doenças como hepatite B, C e HIV (ALMEIDA MCM, et al., 2015, RODRIGUES OS, et al., 2017).

\section{CONCLUSÃO}

Foi possível, através do presente estudo, identificar riscos ocupacionais entre profissionais de enfermagem que atuam em unidade de terapia intensiva, e evidenciar que, independentemente, dos riscos serem físicos, químicos, ergonômicos, biológicos, de acidentes ou psicossociais, esses podem influenciar de forma direta na qualidade da assistência à saúde do paciente, pois contribuem de forma negativa nas condições de saúde dos trabalhadores, podendo levar ao processo de adoecimento. Através dessa pesquisa, foi visto como o ambiente laboral pode levar os profissionais a exposição de diversos riscos, necessitando de adoção de medidas de biossegurança e precaução padrão, de modo a minimizar os riscos de acidentes ou doenças relacionadas ao trabalho durante os cuidados que são prestados de forma direta e contínua a pacientes em estado crítico. 


\section{REFERÊNCIAS}

1. AIRES RKD, et al. Assistência de enfermagem às manifestações emocionais ao familiar do paciente de uma unidade de terapia intensiva.BrazilianJournalofDevelopment, 2020; 6(3): 16267-16279.

2. ALMEIDA MCM, et al. Seguimento clínico de profissionais e estudantes da área da saúde expostos a material biológico potencialmente contaminado. Revista da Escola de Enfermagem da USP, 2015; 49(2): 261-266.

3. ARAGÃO JA, et al. Exposição ocupacional a fluidos biológicos em acidentes com perfurocortantes na equipe de enfermagem hospitalar. Enferm. Foco, 2019; 10(1): 58-64.

4. BALSANELLI AP, CUNHA ICKO. Liderança do enfermeiro em unidade de terapia intensiva e sua relação com ambiente de trabalho. Rev. Latino-Am. Enfermagem, 2015; 23(1):106-113.

5. BRASIL. Conselho Nacional de Saúde. Comissão Nacional de Ética em Pesquisa. Resolução № 466/2012. Diário Oficial da União. Brasília, DF, 2012.

6. BRASIL. Conselho Nacional de Saúde. Comissão Nacional de Ética em Pesquisa. Resolução № 510/2016. Diário Oficial da União. Brasília, DF, 2016.(a).

7. BRASIL. Conselho Federal de Enfermagem. COFEN. Resolução No 527/2016.(b).

8. CANALLI RTC. Riscos Ocupacionais e Acidentes com Material Biológico em Profissionais de Enfermagem da Saúde Coletiva.Tese (Doutorado em Enfermagem Fundamental) - Escola de Enfermagem de Ribeirão Preto da Universidade de São Paulo, 2012.

9. CARRARA GLR, et al. Riscos ocupacionais e os agravos à saúde dos profissionais de enfermagem. Revista Fafibe On-Line, 2015; 8(1): 265-286.

10. FONTANA RT, LAUTERT L. A situação de trabalho da enfermagem e os riscos ocupacionais na perspectiva da ergologia. Rev. Latino-Am. Enfermagem nov.-dez. 2013;21(6):1306-13.

11. ILAPA-RODRIGUEZ EO, et al. Medidas para adesão às recomendações de biossegurança para a equipe de enfermagem. Enfermería Global, 2018; 17(49): 36-67.

12. LUIZE PB, et al. Condutas após exposição ocupacional a material biológico em um hospital especializado em oncologia. Texto \& Contexto Enfermagem, 2015; 24(1): 170-177.

13. MELO FMS, et al. Conhecimentos de enfermeiros sobre acidentes de trabalho. Revista da Rede de Enfermagem do Nordeste, 2017; 18(2): 173-80.

14. MORAES KKO, et al. Exposição da equipe de enfermagem aos riscos biológicos em unidade de terapia intensiva: revisão integrativa. Revista Inova Saúde,2016; (5)2: 163-184.

15. OLIVEIRA MM, et al. Riscos ocupacionais e suas repercussões nos profissionais de enfermagem no âmbito hospitalar.Revista Enfermagem Contemporânea, 2017; 6(2): 129-138.

16. PADILHA KG, et al. Carga de trabalho de enfermagem, estresse/burnout, satisfação e incidentes em unidade de terapia intensiva de trauma. Texto Contexto Enferm, 2017; 26(3): p.2-8.

17. PASA TS, et al. Riscos ergonômicos para trabalhadores de enfermagem ao movimentar e remover pacientes. RevEnferm UFSM, 2015; 5(1): 92-102.

18. PASCOAL KPMF, et al. Avaliação da qualidade de vida, estresse e saúde mental dos profissionais de saúde das unidades de terapia intensiva. Revista Interdisciplinar em Saúde, 2019; 6(5): 19-30.

19. PIMENTA AM, ASSUNÇÃO AA. Estresse no trabalho e hipertensão arterial em profissionais de enfermagem da rede municipal de saúde de Belo Horizonte, Minas Gerais, Brasil. Revista Brasileira de Saúde Ocupacional, 2016; 41(6): 1-11.

20. PORTELA NLC, et al. Riscos ocupacionais entre profissionais de enfermagem: revisão integrativa da literatura. Revista Ciência e Saberes, 2015;1(1): 81-85.

21. RIBEIRO IAP, et al. Biossegurança na unidade de terapia intensiva: uma revisão bibliográfica. Revista UNINGÁ, 2017; 54(1):20-28

22. RODRIGUES MA, et al. Divergências entre legislações do dimensionamento de enfermagem em unidades de terapia intensiva. Enferm. Foco, 2017; 8(1): 12-16.

RODRIGUES PS, et al. Acidente ocupacional entre profissionais de enfermagem atuantes em setores críticos de um pronto-socorro. Esc. Anna Nery,2017; 21(2):20170040.

23. SANTOS SS, et al. Síndrome de Burnout em enfermeiros de unidade de terapia intensiva: produção científica de enfermagem. Revista Destaques Acadêmicos, 2018; 10(3): 190-198.

24. SILVA C, BATISTA EC. Estresse ocupacional em enfermeiros e técnicos de enfermagem intensivistas de uma UTI adulto. Revista Interdisciplinar, 2017; 10(1): 118-128.

25. VASCONCELOS EM, MARTINO MMF. Preditores da síndrome de burnout em enfermeiros de unidade de terapia intensiva. Revista Gaúcha de Enfermagem, 2017; 38(4): 65354. 